\title{
Modeling the Anisotropic Reflectance of a Surface with Microstructure Engineered to Obtain Visible Contrast after Rotation
}

Luongo, Andrea; Falster, Viggo; Doest, Mads Emil Brix; Li, Dongya; Regi, Francesco; Zhang, Yang; Tosello, Guido; Nielsen, Jannik Boll; Aanæs, Henrik; Frisvad, Jeppe Revall

\section{Published in:}

Proceedings of the ICCV 2017 International Conference on Computer Vision

Link to article, DOI:

10.1109/ICCVW.2017.27

Publication date:

2017

Document Version

Peer reviewed version

Link back to DTU Orbit

Citation (APA):

Luongo, A., Falster, V., Doest, M. E. B., Li, D., Regi, F., Zhang, Y., Tosello, G., Nielsen, J. B., Aanæs, H., \& Frisvad, J. R. (2017). Modeling the Anisotropic Reflectance of a Surface with Microstructure Engineered to Obtain Visible Contrast after Rotation. In Proceedings of the ICCV 2017 International Conference on Computer Vision (pp. 159-165). IEEE. https://doi.org/10.1109/ICCVW.2017.27

\section{General rights}

Copyright and moral rights for the publications made accessible in the public portal are retained by the authors and/or other copyright owners and it is a condition of accessing publications that users recognise and abide by the legal requirements associated with these rights.

- Users may download and print one copy of any publication from the public portal for the purpose of private study or research.

- You may not further distribute the material or use it for any profit-making activity or commercial gain

- You may freely distribute the URL identifying the publication in the public portal 


\title{
Modeling the Anisotropic Reflectance of a Surface with Microstructure Engineered to Obtain Visible Contrast after Rotation
}

\author{
Andrea Luongo, Viggo Falster, Mads Brix Doest, Dongya Li, Francesco Regi, Yang Zhang, \\ Guido Tosello, Jannik Boll Nielsen, Henrik Aanæs, Jeppe Revall Frisvad \\ Technical University of Denmark \\ aluo@dtu.dk
}

\begin{abstract}
Engineering of surface structure to obtain specific anisotropic reflectance properties has interesting applications in large scale production of plastic items. In recent work, surface structure has been engineered to obtain visible reflectance contrast when observing a surface before and after rotating it 90 degrees around its normal axis. We build an analytic anisotropic reflectance model based on the microstructure engineered to obtain such contrast. Using our model to render synthetic images, we predict the above mentioned contrasts and compare our predictions with the measurements reported in previous work. The benefit of an analytical model like the one we provide is its potential to be used in computer vision for estimating the quality of a surface sample. The quality of a sample is indicated by the resemblance of camera-based contrast measurements with contrasts predicted for an idealized surface structure. Our predictive model is also useful in optimization of the microstructure configuration, where the objective for example could be to maximize reflectance contrast.
\end{abstract}

\section{Introduction}

Engineering of surface microstructure to obtain custom reflectance properties, or so-called appearance printing, has many applications in product design and manufacturing. This research area has received significant attention [23, 11, 10, 7, 8, 14] and, recently, tooling was done with the objective of inserting a simple anisotropic surface microstructure into economic manufacturing processes [9]. The intended functionality of the anisotropic surface microstructure was to obtain high reflectance contrast for the surface when viewed from above at orthogonal angles. Using a microscope and a camera, the contrast was measured for different surface structure configurations to find the configuration revealing highest contrast [16].

In this work, we build an analytic bidirectional re-

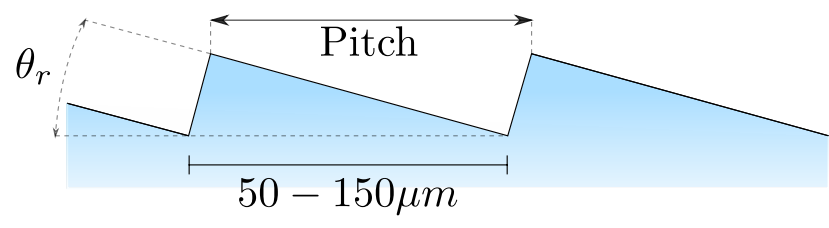

Figure 1. Engineered surface microstructure used by previous authors [9, 16] to obtain reflectance contrast when the surface is observed from above at orthogonal angles. We build an analytic BRDF model for this type of surface.

flectance distribution function (BRDF) that models the anisotropic reflectance properties of the functional surface tested by previous authors [9, 16]. Our analytic BRDF model has two benefits. It is (1) useful for finding the surface structure configuration that theoretically produces optimal contrast. It also (2) enables estimation of surface quality from photographs. As an example, deviation in the contrast measured for a physical sample from the contrast predicted for an idealized surface corresponds to surface quality deviation, and contrast is measurable using simple computer vision [16].

The simple anisotropic microstructure that produces visible contrast when viewed at orthogonal angles is created by having a sequence of small, parallel ridges as shown in Figure 1 . The angle $\theta_{r}$ is a microstructure configuration referred to as the ridge angle. Based on how the structure is rotated around its macrosurface normal, it reflects light in different ways. Thus contrast can be generated by rotating the object by $90^{\circ}$.

The reflectance properties of the ridged surface have only been studied experimentally [16]. The analytic BRDF we provide is built for this particular ridged surface structure, but a similar procedure could be used to derive an analytical model for a surface with a different engineered surface structure. Figure 2 shows an example of a quad rendered with our BRDF before and after rotation by $90^{\circ}$. The contrast produced by the ridges having orthogonal orientation is clearly visible in this image. 


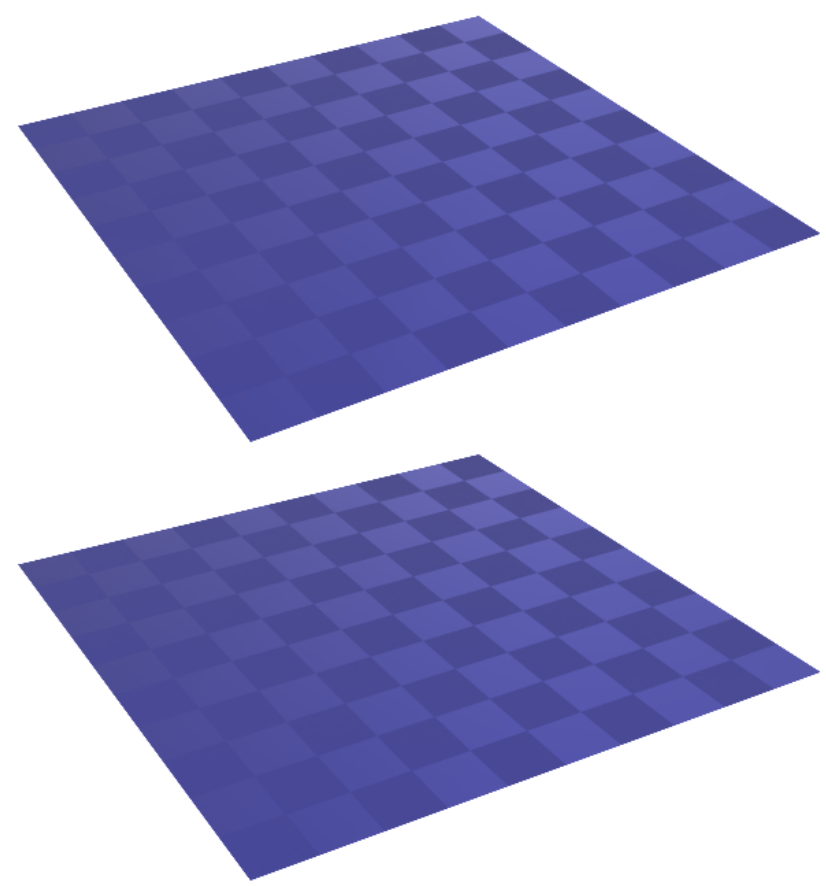

Figure 2. Quad rendered using our BRDF model before and after rotation by $90^{\circ}$. The orientation of the ridges follows a checkerboard pattern: two adjacent squares have ridges oriented in orthogonal directions.

\section{Related Work}

The work of Torrance and Sparrow [18, 19] is an early example of measuring the reflectance properties of a surface and subsequently developing a BRDF model for predicting the measured properties. Torrance and Sparrow [18] investigated metals and ceramics and processed the surfaces of their samples with the objective of having isotropic, random rough surfaces. They developed their BRDF model for this type of surface in order to explain surprising occurrences of off-specular peaks in the reflectance measurements [19]. Our work is similar, but we model the reflectance properties of plastic samples with an anisotropic, ridged surface.

With a similar approach, Ward [22] measured and modeled the bidirectional reflectance properties of anisotropic, random rough surfaces. He found good agreement between model and measurements for materials such as varnished wood and unfinished (rolled) or brushed metals. Our work is different in the sense that we model a ridged rough surface instead of a random rough surface.

Poulin and Fournier [15] presented one of the first BRDF models for an anisotropic surface with a specific microstructure. Their model assumes a microstructure consisting of half cylinders each with its axis lying in the surface tangent plane. More generically, Ashikhmin et al. [2] describe a methodology for generating a BRDF according to a given microfacet normal distribution function. Our ridged surface microstructure requires a slightly different approach as the microfacet normals are predominantly in two directions. The specific microstructure we model is interesting because it can be engineered. This enables us to compare reflectance properties predicted by our model with measured reflectance properties.

Using a generic BRDF model [2], it is possible to match observed reflectance properties by acquiring spatially varying microfacet normal distribution functions for an anisotropic surface [21]. This is impressive, but then deviations between predicted and measured properties cannot be used to assess how close an engineered microstructure is to the desired idealized microstructure.

Researchers working with techniques for BRDF printing have an opportunity to compare predicted reflectance properties with those of engineered surfaces. Weyrich et al. [23] use micro milling to obtain a surface structure with a specific microfacet normal distribution. This is similar to the tooling part of the manufacturing process that we are modeling [9]. Our added step of transferring the tool microstructure to a polymer component enables absorption (colored surfaces). In previous work [11, 7], absorption was added by applying different inks to the surface. This means that their BRDF model is a weighted average of differently oriented ink BRDFs, where we have a combination of surface and subsurface scattering effects. Other authors [10, 8] improve the microstructure resolution as compared with Weyrich et al. [23], but their techniques do not allow for absorption effects. In the work of Levin et al. [8], microfacets are at a scale that requires a BRDF model based on wave optics. Our pitches ranging from 50 to 150 microns can safely be modeled using geometrical optics. None of this previous work on BRDF printing includes shadowing and masking in their BRDF models. This is however important in the microstructure we investigate due to the steep slope of the ridge edges.

In recent work, Pereira et al. [14] show that magnetic microflakes can be used for anistropic BRDF printing. They measure the BRDFs printed by their technique but do not provide a predictive BRDF model.

Levin et al. [8] investigated the same kind of contrast that we are aiming at with our ridged surface structure. Their technique is however very different as it is based on wave interference effects. While they seem to achieve better contrast than ridged surfaces, they use photolithography which has high capital and operational cost and cannot easily be used with polymeric or curved substrates [1]. Nevertheless, it is noteworthy how easily their very small surface features produce contrast through wave interference.

McGunnigle [12] uses a bivariate Gaussian distribution (no Fresnel or geometrical attenuation effects) to model the anisotropic reflectance of a surface sandpapered in one direction. While his directional surface microstructure seems 


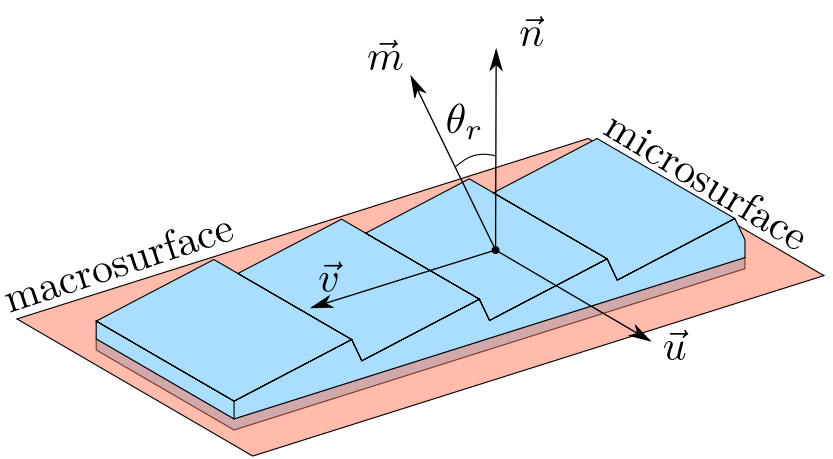

Figure 3. Microstructured surface and simplified macrosurface.

a bit like ours, his reflectance model is one-dimensional and considers only the azimuthal angle of the light source.

\section{BRDF Model}

The engineered surface that we consider is composed of many parallel ridges with a pitch of between $50 \mu \mathrm{m}$ and $150 \mu \mathrm{m}$ (see Figure 1). If viewed at a reasonable distance, these details can be assumed to be too small to be seen directly (at a distance of $0.5 \mathrm{~m}$, humans can discern details of about $150 \mu \mathrm{m} \mathrm{[13]).} \mathrm{Thus} \mathrm{we} \mathrm{choose} \mathrm{to} \mathrm{model} \mathrm{our} \mathrm{sur-}$ face by a macrosurface with an appropriate BRDF. In this way, the details of the microstructure are represented by the reflectance properties of the surface. This is analogous to other microfacet BRDF models [19, 3, 4, 2], where a rough surface with a complicated microgeometry is modeled by a simpler surface with an appropriate BRDF that can replicate the overall light scattering of the microsurface.

Figure 3 illustrates the macrosurface for our particular microsurface. In this model, we have a microsurface normal $\vec{m}$ and a macrosurface normal $\vec{n}$ (both are unit vectors). In addition, $\vec{u}$ is a vector parallel to the ridges and orthogonal to the normal $\vec{n}$, and $\vec{v}$ is a vector aligned with the direction of the pitches. Together, $\vec{u}, \vec{v}, \vec{n}$ is an orthonormal basis of the macrosurface tangent space.

Microfacet models represent the microsurface in terms of a microfacet reflectance function $f_{m}$, a geometrical attenuation function $G$, and a normal distribution function $D$. These are combined by integration over all microfacet normals to form a BRDF $f$ for the macrosurface [20].

\subsection{Geometrical Attenuation Function $G$}

The portion of the microsurface with normal $\vec{m}$ visible from both directions $\vec{\omega}_{i}$ and $\vec{\omega}_{o}$ is described by the geometrical attenuation function $G\left(\vec{\omega}_{i}, \vec{\omega}_{o}, \vec{m}\right)$. This means that the function models shadowing and masking effects.

An exact formulation of $G$ is rarely available since it depends on the geometrical details of the particular surface. Most often, the function is approximated based on assumptions about the surface geometry (such as v-grooves [19]).

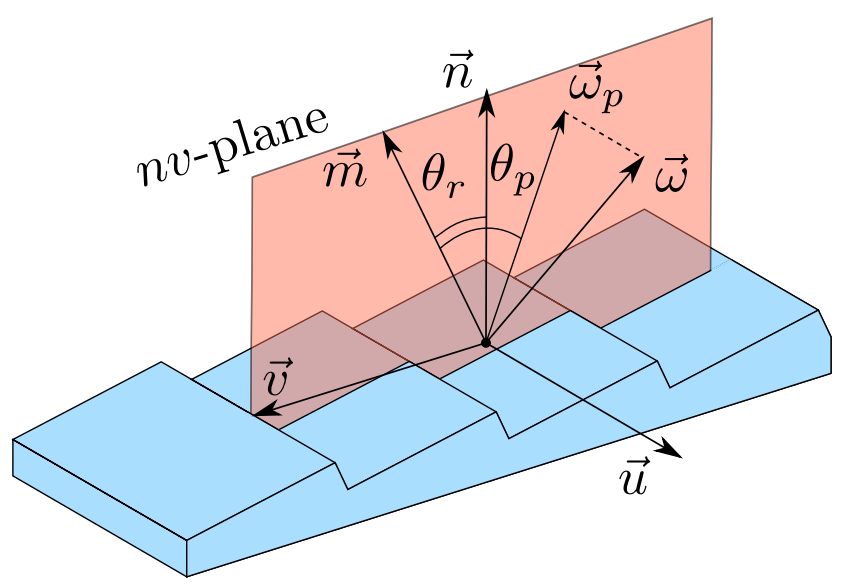

Figure 4 . The angle $\theta_{p}$ between the microsurface normal $\vec{m}$ and the projection of the vector $\vec{\omega}$ on the $n v$-plane is used to evaluate the geometrical attenuation function.

Smith [17] derived an approximation of $G$ for surfaces with Gaussian microfacet normal distribution. This has the useful property of being separable into the product of two mono-directional functions (one for shadowing and one for masking):

$$
G\left(\vec{\omega}_{i}, \vec{\omega}_{o}, \vec{m}\right) \approx G_{1}\left(\vec{\omega}_{i}, \vec{m}\right) G_{1}\left(\vec{\omega}_{o}, \vec{m}\right)
$$

Given the particular regularity of the microsurface we are dealing with, we have derived an expression for $G_{1}$ (details are provided in Appendix A) that is suitable for our model:

$$
\begin{aligned}
G_{1}(\vec{\omega}, \vec{m})= & \chi^{+}\left(\frac{\vec{\omega}_{p} \cdot \vec{m}}{\vec{\omega}_{p} \cdot \vec{n}}\right) \\
& \times\left[1-\min \left(1,\left|\tan \theta_{r} \tan \theta_{p}\right|\right)\right],
\end{aligned}
$$

where $\chi^{+}(a)$ denotes a Heaviside step function that is 1 for $a>0$ and 0 otherwise. We let $\theta_{p}$ denote the angle between $\vec{m}$ and the projection $\vec{\omega}_{p}$ of $\vec{\omega}$ on the plane spanned by $\vec{n}$ and $\vec{v}$, see Figure 4 . Thus,

$$
\cos \theta_{p}=\frac{\vec{\omega}_{p} \cdot \vec{m}}{\left|\vec{\omega}_{p}\right|}=\frac{(\vec{\omega}-(\vec{\omega} \cdot \vec{u}) \vec{u}) \cdot \vec{m}}{|\vec{\omega}-(\vec{\omega} \cdot \vec{u}) \vec{u}|},
$$

which reveals that the orientation of the macrosurface is required to evaluate the geometrical attenuation function. This is as expected since we are dealing with an anisotropic surface microstructure.

\subsection{Microfacet Distribution Function $D$}

The manufacturing process introduces irregularities on the ridges. The surface microstructure of physical samples is therefore not as regular as the idealized surface illustrated in Figure 1. In reality, it is rather rough as illustrated in Figure 5. Roughnesses have been measured for physical samples in previous work using optical profilometry [9, 16]. 


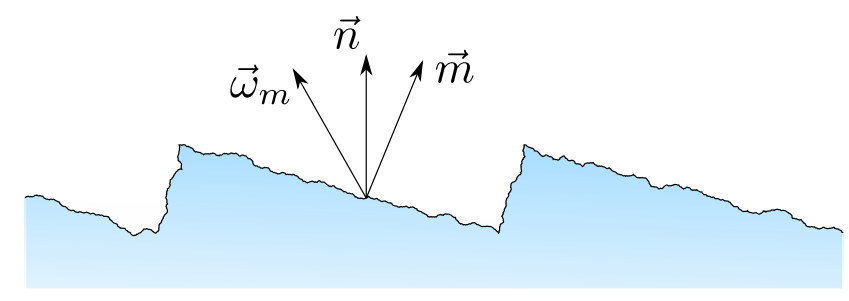

Figure 5. Rough surface.

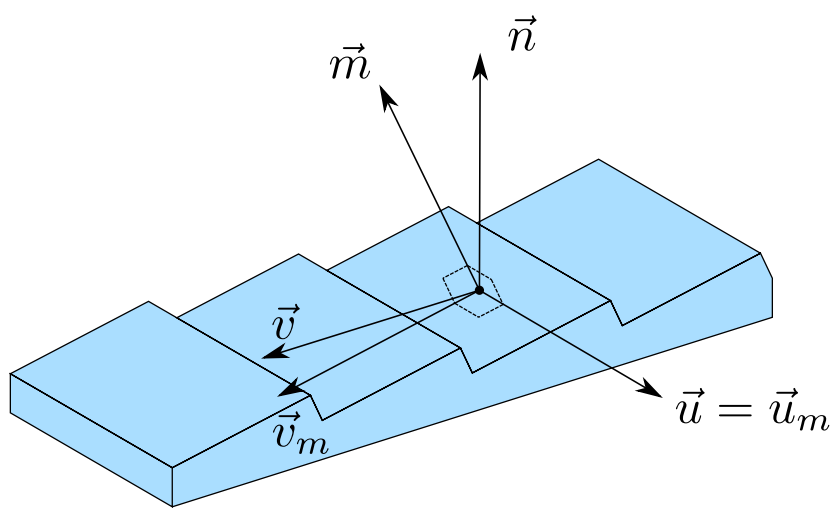

Figure 6. Orthonormal basis formed by $\vec{u}_{m}, \vec{v}_{m}, \vec{m}$.

These measurements reveal that the ridges are certainly not smooth. Thus, at a given point $\boldsymbol{x}$ on the microsurface the normal $\vec{\omega}_{m}$ is usually slightly different from the pitch normal $\vec{m}$.

The distribution of normals $D\left(\vec{\omega}_{m}\right)$ statistically describes the orientation of these irregularities across the microsurface. Many microfacet distribution functions have been defined over the years [19, 3, 4, 20]. In order to highlight the anisotropic nature of the surface we are working with, we use the anisotropic Beckmann distribution function [5], which is defined by

$$
\begin{aligned}
& D\left(\vec{\omega}_{m}\right)=\frac{\chi^{+}\left(\vec{\omega}_{m} \cdot \vec{m}\right)}{\pi \alpha_{u} \alpha_{v} \cos ^{4}\left(\theta_{m}\right)} \\
& \quad \times \exp \left(-\tan ^{2} \theta_{m}\left(\frac{\cos ^{2} \phi_{m}}{\alpha_{u}^{2}}+\frac{\sin ^{2} \phi_{m}}{\alpha_{v}^{2}}\right)\right) .
\end{aligned}
$$

This distribution function is centred around the pitch normal $\vec{m}$, and the parameters $a_{u}$ and $a_{v}$ represent the stretching coefficients of the distribution along the $\vec{u}_{m}$ and $\vec{v}_{m}$ directions, respectively, see Figure 6 Together, $\vec{u}_{m}, \vec{v}_{m}, \vec{m}$ form an orthonormal basis and the microsurface normal $\vec{w}_{m}$ can be written in spherical coordinates as

$$
\begin{aligned}
\vec{\omega}_{m}= & \sin \left(\theta_{m}\right) \cos \left(\phi_{m}\right) \vec{u}_{m} \\
& +\sin \left(\theta_{m}\right) \sin \left(\phi_{m}\right) \vec{v}_{m}+\cos \left(\theta_{m}\right) \vec{m}
\end{aligned}
$$

\subsection{Macrosurface and Microfacet BRDFs}

The normal distribution function $D\left(\vec{\omega}_{m}\right)$ and the geometrical attenuation function $G\left(\vec{\omega}_{i}, \vec{\omega}_{o}, \vec{m}\right)$ are combined into a macrosurface BRDF using [20]

$$
\begin{aligned}
f_{M}\left(\boldsymbol{x}, \vec{\omega}_{i}, \vec{\omega}_{o}\right)= & \int f_{m}\left(\vec{\omega}_{i}, \vec{\omega}_{o}, \vec{\omega}_{m}\right) D\left(\vec{\omega}_{m}\right) G\left(\vec{\omega}_{i}, \vec{\omega}_{o}, \vec{\omega}_{m}\right) \\
& \times\left|\frac{\vec{\omega}_{i} \cdot \vec{\omega}_{m}}{\vec{\omega}_{i} \cdot \vec{n}}\right|\left|\frac{\vec{\omega}_{o} \cdot \vec{\omega}_{m}}{\vec{\omega}_{o} \cdot \vec{n}}\right| d \vec{\omega}_{m}
\end{aligned}
$$

For the microfacet BRDF $f_{m}$, we assume that a microfacet is smooth so that it reflects and refracts light as a perfectly specular material. Reflection is described by one BRDF $f_{m}^{r}$ and some of the refracted light returns due to subsurface scattering. We approximate this part by another $\operatorname{BRDF} f_{m}^{s s}$. The function $f_{m}$ is then defined by

$$
f_{m}\left(\vec{\omega}_{i}, \vec{\omega}_{o}, \vec{\omega}_{m}\right)=f_{m}^{r}\left(\vec{\omega}_{i}, \vec{\omega}_{o}, \vec{\omega}_{m}\right)+f_{m}^{s s}\left(\vec{\omega}_{i}, \vec{\omega}_{o}, \vec{\omega}_{m}\right)
$$

These BRDFs are based on a directional Dirac deltafunction $\delta$ (just like the BRDF of a perfect mirror). We use Fresnel reflectance $F_{r}$ as the specular reflectance and include a change of coordinates to enable integration over microfacet normals [20]. We then have

$$
f_{m}^{r}\left(\vec{\omega}_{i}, \vec{\omega}_{o}, \vec{\omega}_{m}\right)=F_{r}\left(\vec{\omega}_{i}, \vec{\omega}_{m}\right) \frac{\delta\left(\vec{\omega}_{h}, \vec{\omega}_{m}\right)}{4\left(\vec{\omega}_{i} \cdot \vec{\omega}_{h}\right)^{2}}
$$

where $\vec{\omega}_{h}=\left(\vec{\omega}_{o}+\vec{\omega}_{i}\right) /\left|\vec{\omega}_{o}+\vec{\omega}_{i}\right|$ is the half vector of reflection.

Although subsurface scattering happens for many BRDF inputs, we limit our model to only include subsurface scattering of the light that was lost to refraction in the reflection case. This light is certainly missing and including it is a first step. This makes our model similar to the BRDF approximation of subsurface scattering described by Jensen et al. [6]. We have

$$
f_{m}^{s s}\left(\vec{\omega}_{i}, \vec{\omega}_{o}, \vec{\omega}_{m}\right)=F_{t}\left(\vec{\omega}_{i}, \vec{\omega}_{m}\right) F_{t}\left(\vec{\omega}_{o}, \vec{\omega}_{m}\right) \frac{\rho_{d}}{\pi} \frac{\delta\left(\vec{\omega}_{h}, \vec{\omega}_{m}\right)}{4\left(\vec{\omega}_{i} \cdot \vec{\omega}_{h}\right)^{2}},
$$

where $F_{t}=1-F_{r}$ is the Fresnel transmittance, and $\rho_{d}$ is the diffuse reflectance of the material.

By inserting Eq. 5 into Eq. 4, we arrive at our macrosurface BRDF:

$$
f\left(\boldsymbol{x}, \vec{\omega}_{i}, \vec{\omega}_{o}\right)=f_{r}\left(\boldsymbol{x}, \vec{\omega}_{i}, \vec{\omega}_{o}\right)+f_{s s}\left(\boldsymbol{x}, \vec{\omega}_{i}, \vec{\omega}_{o}\right),
$$

where the reflection term is

$$
f_{r}\left(\boldsymbol{x}, \vec{\omega}_{i}, \vec{\omega}_{o}\right)=\frac{F_{r}\left(\vec{\omega}_{i}, \vec{\omega}_{h}\right)}{4\left|\vec{\omega}_{i} \cdot \vec{n}\right|\left|\vec{\omega}_{o} \cdot \vec{n}\right|} G\left(\vec{\omega}_{i}, \vec{\omega}_{o}, \vec{\omega}_{h}\right) D\left(\vec{\omega}_{h}\right)
$$

and the subsurface scattering term is

$$
\begin{aligned}
f_{s s}\left(\boldsymbol{x}, \vec{\omega}_{i}, \vec{\omega}_{o}\right)= & \frac{\rho_{d}}{\pi} F_{t}\left(\vec{\omega}_{o}, \vec{\omega}_{h}\right) F_{t}\left(\vec{\omega}_{i}, \vec{\omega}_{h}\right) \\
& \times \frac{G\left(\vec{\omega}_{i}, \vec{\omega}_{o}, \vec{\omega}_{h}\right) D\left(\vec{\omega}_{h}\right)}{4\left|\vec{\omega}_{i} \cdot \vec{n}\right|\left|\vec{\omega}_{o} \cdot \vec{n}\right|} .
\end{aligned}
$$



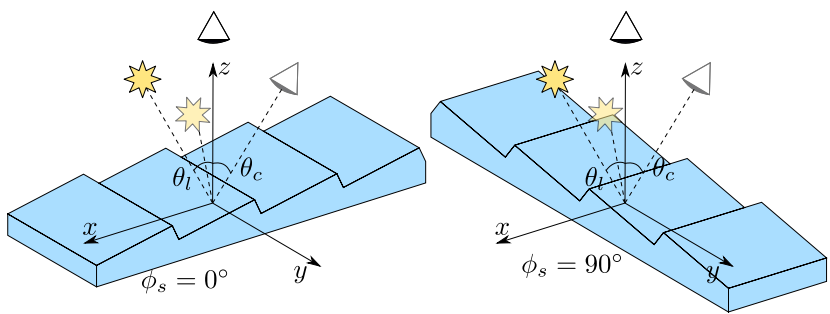

Figure 7. Configuration of the experiment for measuring contrast.

\section{Experiments}

We test our model by investigating its ability to predict the contrast measurements by Regi et al. [16]. These were conducted by photographing the surface before and after rotating the microstructure $90^{\circ}$ around its macrosurface normal axis. Figure 7 illustrates the configuration of this experiment. They observed the samples with a digital microscope modified to hold an LED light source at a fixed position relative to the camera so that the angle between the camera and the light source was constant: $\theta_{l}=10^{\circ}$.

The parameters considered in the experiment are: the ridge angle $\theta_{r}$ which could assume the values $5^{\circ}, 10^{\circ}, 15^{\circ}$, and $20^{\circ}$; the camera tilting angle $\theta_{c}$ with values $-20^{\circ}$, $-10^{\circ}, 0^{\circ}, 10^{\circ}$, and $20^{\circ}$, and the azimuthal angle of rotation of the structure $\phi_{s}$ with values $0^{\circ}, 90^{\circ}$, and $180^{\circ}$. The radiant exposure was measured under constant lighting conditions and varying parameters. The contrast was then evaluated as the difference between the measurements at positions $0^{\circ}$ and $90^{\circ}$ and between $90^{\circ}$ and $180^{\circ}$ for $\phi_{s}$.

To predict these contrast measurements, we reproduced the same settings in a rendering framework and measured the radiant exposure $\left[\frac{\mathrm{J}}{\mathrm{m}^{2}}\right]$ (up to an unknown scaling factor $k$ ). Renderings were based on the BRDF described in the previous section and we compare our contrast measurements with the results presented by Regi et al. [16] in the following section.

\section{Results}

Our contrast predictions are compared with the measured contrasts in Figure 8. The mean contrast was evaluated by keeping one parameter constant and averaging all the contrasts obtained by varying the other parameters.

As in the measurements, we find maximum contrast for zero tilting angle $\left(\theta_{c}=0^{\circ}\right)$ and decreasing contrast when the camera is tilted. We also find that the anisotropic structure of the surface makes the contrast between the azimuthal angles $0^{\circ}$ and $90^{\circ}$ stronger than the contrast between $90^{\circ}$ and $180^{\circ}$. With respect to the ridge angle $\theta_{r}$, our model predicts the highest contrast with a $5^{\circ}$ angle. This is theoretically plausible as a five degrees ridge angle should leave most microfacets with a normal so that light is reflected in the macrosurface normal direction when $\theta_{c}=0^{\circ}$.
Camera angle, $\theta_{c} \quad$ Ridge angle, $\theta_{r} \quad$ Surface angle, $\phi_{s}$
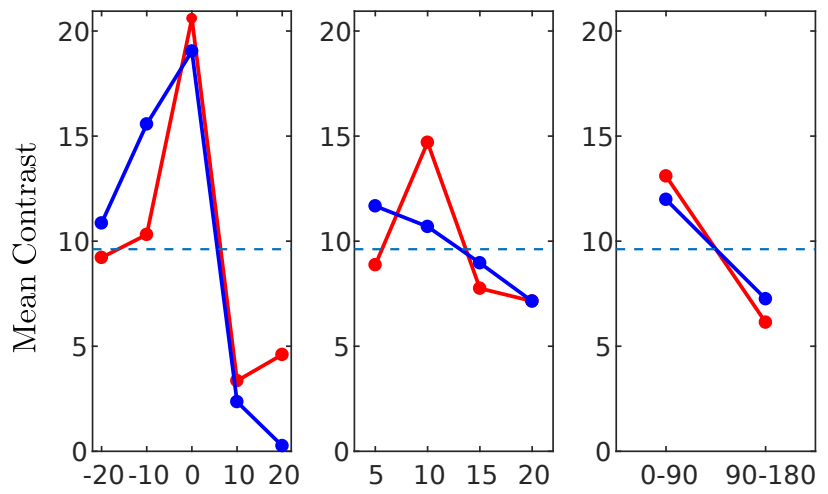

Figure 8. Comparison of mean contrasts for different values of the parameters $\theta_{c}, \theta_{r}$, and $\phi_{s}$. Measured contrasts [16] are in red and our predicted contrasts are in blue.

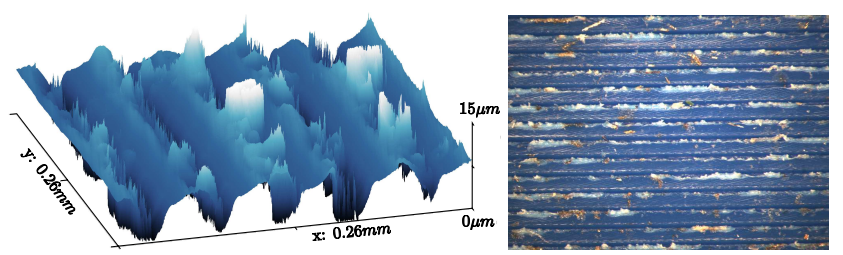

Figure 9. Small part of a manufactured sample. Visualization of a height map acquired with an industrial laser confocal microscope (left) and a microscope image (right).

The most significant difference between prediction and measurement is that measurements found highest contrast for a ridge angle of $\theta_{r}=10^{\circ}$. We think that this result might be caused by the presence of noise in the surface structure due to the manufacturing process. To support this conjecture, we have produced samples similar to the ones in previous work [9, 16] and investigated the microstructure of the tool and the manufactured plastic sample. Figure 9 shows a 3D visualization of height data captured with a $3 \mathrm{D}$ laser confocal scanner and a microscope image both of the plastic surface. While the original surfaces produced by Regi et al. [16] may have been higher quality, there is no doubt that the manufacturing process produces inaccuracies both in the tool and in the sample microstructure. In the tool, we have observed small burrs, especially along ridge edges. These burrs have a tendency to leave residues of material on the surface and create substantial artifacts. The white bulky peaks in Figure 9 are examples of such artifacts. These imperfections in the surface become more significant for small ridge angles and may easily hide the signal from the ridged structure in noise. We believe this is a plausible explanation for this deviation between prediction and measurement.

\section{Discussion and Future Work}

We have developed a new model for predicting the reflectance properties of an engineered anisotropic surface 


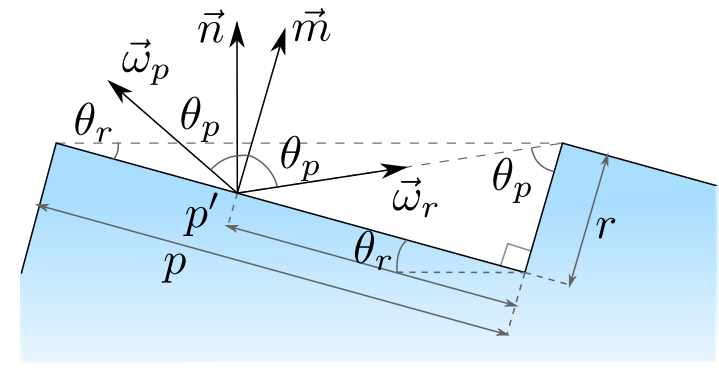

Figure 10. The surface fraction masked by the ridged structure is given by the ratio between $p$ and $p^{\prime}$.

made of parallel micro ridges. Our model provides a BRDF based on microfacet theory including an expression for the geometrical attenuation function. The BRDF describes our particular type of ridged surface, but a similar procedure could be employed to model other engineered surface microstructures. We validated our model by comparing with experimental measurements from previous work. Our results are quite similar to the measurements, but we observed some deviations. If deviations are due to manufacturing artifacts, as we conjecture, our model is useful as a tool for computer vision based quality inspection of optical functional surfaces of this kind. In addition, our model provides many opportunities for optimizing surface structure with the objective of maximizing contrast, for example. It is significantly easier to modify microstructure configuration in simulation as compared with experiment.

In the future, we would like to further support our conjecture that contrast measurements converge to predicted contrasts as sample quality improves. This will be investigated as tooling and manufacturing processes improve to provide higher quality samples. Moreover, comparison of anisotropic BRDF measurements with predicted values would also be interesting as an alternative to the more overall contrast measurements.

\section{A. The $G_{1}$ Function for a Ridged Surface}

This appendix provides some details about the derivation of the geometrical attenuation function described in Eq.2

The value of the function $G_{1}$ is given by the ratio between the portion of the pitch surface visible from a given direction $\vec{\omega}_{p}$ and the total pitch surface. Figure 10 provides some elements that are useful for the derivation of Eq. 2 . The vector $\vec{\omega}_{r}$ represents the reflection of $\vec{\omega}_{p}$ around the surface normal $\vec{m}, \theta_{r}$ is the ridge angle and $\theta_{p}$ is the angle between $\vec{\omega}_{p}$ and $\vec{m}, p$ and $r$ represent respectively the length of the pitch and the length of the ridge, and $p^{\prime}$ represents the length of the portion of pitch surface for which the reflection vector $\vec{\omega}_{r}$ is blocked by the ridge.

Now, $G_{1}$ is described by

$$
G_{1}\left(\vec{\omega}_{p}, \vec{m}\right)=1-\frac{p^{\prime}\left(\vec{\omega}_{p}, \vec{m}\right)}{p},
$$

and the value of $p^{\prime}$ is

$$
p^{\prime}\left(\vec{\omega}_{p}, \vec{m}\right)=r \tan \theta_{p}=p \tan \theta_{r} \tan \theta_{p} .
$$

Then, by inserting Eq. 8 into Eq. 7, we have

$$
G_{1}\left(\vec{\omega}_{p}, \vec{m}\right)=1-\tan \theta_{r} \tan \theta_{p} .
$$

Since the value of $p^{\prime}$ might become greater than $p$ for certain combinations of angles $\theta_{r}$ and $\theta_{p}$, we modify Eq.9 9 and get

$$
\begin{aligned}
G_{1}\left(\vec{\omega}_{p}, \vec{m}\right)= & \chi^{+}\left(\frac{\vec{\omega}_{p} \cdot \vec{m}}{\vec{\omega}_{p} \cdot \vec{n}}\right) \\
& \times\left[1-\min \left(1,\left|\tan \theta_{r} \tan \theta_{p}\right|\right)\right] .
\end{aligned}
$$

In a similar way, it can be shown that for an arbitrary direction $\vec{\omega}$ not lying in the plane spanned by the $\vec{n}$ and $\vec{m} \mathrm{Eq} .10$ is still valid and depends only on the projection vector $\vec{\omega}_{p}$ and the surface normal $\vec{m}$.

\section{References}

[1] C. Acikgoz, M. A. Hempenius, J. Huskens, and G. J. Vancso. Polymers in conventional and alternative lithography for the fabrication of nanostructures. European Polymer Journal, 47(11):2033-2052, November 2011.

[2] M. Ashikmin, S. Premože, and P. Shirley. A microfacetbased BRDF generator. In Proceedings of SIGGRAPH 2000, pages 65-74. ACM/Addison-Wesley, 2000.

[3] J. F. Blinn. Models of light reflection for computer synthesized pictures. Computer Graphics (Proceedings of SIGGRAPH 77), 11(2):192-198, July 1977.

[4] R. L. Cook and K. E. Torrance. A reflectance model for computer graphics. ACM Transactions on Graphics, 1(1):724, January 1982.

[5] E. Heitz. Understanding the masking-shadowing function in microfacet-based brdfs. Journal of Computer Graphics Techniques, 3(2):48-107, June 2014.

[6] H. W. Jensen, S. R. Marschner, M. Levoy, and P. Hanrahan. A practical model for subsurface light transport. In Proceedings of SIGGRAPH 2001, pages 511-518. ACM, August 2001.

[7] Y. Lan, Y. Dong, F. Pellacini, and X. Tong. Bi-scale appearance fabrication. ACM Transactions on Graphics (Proceedings of SIGGRAPH 2013), 32(4):145:1-145:11, July 2013.

[8] A. Levin, D. Glasner, Y. Xiong, F. Durand, W. Freeman, W. Matusik, and T. Zickler. Fabricating BRDFs at high spatial resolution using wave optics. ACM Transactions on Graphics (Proceedings of SIGGRAPH 2013), 32(4):144:1144:13, July 2013. 
[9] D. Li, Y. Zhang, F. Regi, G. Tosello, M. H. Madsen, J. B. Nielsen, H. Aanæs, and J. R. Frisvad. Process chain for fabrication of anisotropic optical functional surfaces on polymer components. In Proceedings of the 17th EUSPEN International Conference, June 2017.

[10] T. Malzbender, R. Samadani, S. Scher, A. Crume, D. Dunn, and J. Davis. Printing reflectance functions. ACM Transactions on Graphics, 31(3):20:1-20:11, May 2012.

[11] W. Matusik, B. Ajdin, J. Gu, J. Lawrence, H. Lensch, F. Pellacini, and S. Rusinkiewicz. Printing spatially-varying reflectance. ACM Transactions on Graphics (Proceedings of SIGGRAPH Asia 2009), 28(5):128:1-128:9, December 2009.

[12] G. McGunnigle. Shape recovery of anisotropic metal surfaces. JOSA A, 26(10):2235-2242, October 2009.

[13] D. Miller, P. Schor, and P. Magnante. Optics of the normal eye. In M. Yanoff and J. S. Duker, editors, Ophthalmology, chapter 2.6, pages 52-60. Mosby/Elsevier, 3rd edition, 2009.

[14] T. Pereira, C. L. Leme, S. Marschner, and S. Rusinkiewicz. Printing anisotropic appearance with magnetic flakes. ACM Transactions on Graphics (Proceedings of SIGGRAPH 2017), 36(4):123:1-123:10, July 2017.

[15] P. Poulin and A. Fournier. A model for anisotropic reflection. In Computer Graphics (Proceedings of SIGGRAPH 90), volume 24, pages 273-282, August 1990.

[16] F. Regi, D. Li, Y. Zhang, J. B. Nielsen, M. H. Madsen, G. Tosello, J. R. Frisvad, and H. Aanæs. A comparison of reflectance properties on polymer micro structured functional surface. In Proceedings of the 17th EUSPEN International Conference, June 2017.

[17] B. Smith. Geometrical shadowing of a random rough surface. IEEE Transactions on Antennas and Propagation, 15(5):668-671, September 1967.

[18] K. E. Torrance and E. M. Sparrow. Off-specular peaks in the directional distribution of reflected thermal radiation. Journal of Heat Transfer, 88(2):223-230, 1966.

[19] K. E. Torrance and E. M. Sparrow. Theory for off-specular reflection from roughened surfaces. Journal of the Optical Society of America, 57(9):1105-1114, September 1967.

[20] B. Walter, S. Marschner, H. Li, and K. Torrance. Microfacet models for refraction through rough surfaces. In Proceedings of Eurographics Symposium on Rendering (EGSR 2007), pages 195-206. The Eurographics Association, 2007.

[21] J. Wang, S. Zhao, X. Tong, J. Snyder, and B. Guo. Modeling anisotropic surface reflectance with example-based microfacet synthesis. In ACM Transactions on Graphics (Proceedings of SIGGRAPH 2008), volume 27, pages 41:1-41:9, August 2008.

[22] G. J. Ward. Measuring and modeling anisotropic reflection. Computer Graphics (Proceedings of SIGGRAPH 92), 26(2):265-272, July 1992.

[23] T. Weyrich, P. Peers, W. Matusik, and S. Rusinkiewicz. Fabricating microgeometry for custom surface reflectance. ACM Transactions on Graphics (Proceedings of SIGGRAPH 2009), 28(3):32:1-32:6, August 2009. 